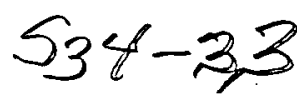

160548

\title{
EMBEDDING IMPEDANCE APPROXIMATIONS IN THE ANALYSIS OF SIS MIXERS
}

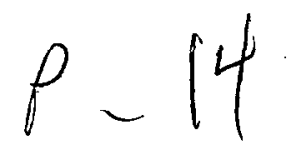

\author{
A. R. Kerr and S.-K. Pan \\ Charlottesville, VA 22903 \\ S. Withington \\ Cavendish Laboratory, University of Cambridge \\ Cambridge CB3 OHE, UK
}

National Radio Astronomy Observatory ${ }^{*} 93-27760$

\begin{abstract}
Future nillimeter-wave radio astronomy instruments will use arrays of many SIS receivers, either as focal plane arrays on individual radio telescopes, or as individual receivers on the many antennas of radio interferometers. Such applications will require broadband integrated mixers without mechanical tuners. To produce such mixers, it will be necessary to improve present mixer design techniques, most of which use the three-frequency approximation to Tucker's quantum mixer theory.
\end{abstract}

This paper examines the adequacy of three approximations to Tucker's theory: (i) the usual three-frequency approximation which assumes a sinusoidal $L$ voltage at the junction, and a short-circuit at all frequencies above the upper sideband, (ii) a five-frequency approximation which allows two $L$ voltage harmonics and five small-signal sidebands, and (iii) a quasi five-frequency approximation in which five small-signal sidebands are allowed, but the $L O$ voltage is assumed sinusoidal. These are compared with a full harmonic-Newton solution of Tucker's equations, including eight $L$ harmonics and their corresponding sidebands, for realistic SIS mixer circuits.

It is shown that the accuracy of the three approximations depends strongly on the value of $\omega R_{H} C$ for the SIS junctions used. For large $\omega R_{H} C$, all three approximations approach the eight-harmonic solution. For $\omega R_{\mathrm{H}} \mathrm{C}$ values in the range 0.5 to 10 , the range of most practical interest, the quasi five-frequency approximation is a considerable improvement over the three-frequency approximation, and should be suitable for much design work. For the realistic SIS mixers considered here, the five-frequency approximation gives results very close to those of the eight-harmonic solution.

Use of these approximations, where appropriate, considerably reduces the computational effort needed to analyze an SIS mixer, and allows the design and optimization of mixers using a personal computer.

"The National Radio Astronomy Observatory is operated by Associated Universities, Inc. under cooperative agreement with the National Science Foundation. 


\section{INTRODUCTION}

The superior sensitivity of SIS mixer receivers at millimeter wavelengths has been clearly demonstrated in recent years [1], and SIS receivers are now used at almost all the world's major millimeter-wave radio astronomy observatories. SIS mixers appear for the most part to be well described by Tucker's quantum theory $[2,3]$, which predicts strong non-classical behavior in a resistive mixer with an extremely sharp $I-V$ nonlinearity. Tucker's theory is normally applied in its three-frequency approximation, in which form it is moderately tractable analytically and allows optimization of designs by small computer.

At present, the most sensitive SIS mixers have one or two mechanical waveguide tuners which allow the RF embedding impedance to be adjusted to suit the particular junction (or array of junctions). While mechanically tuned mixers are acceptable in applications requiring one or two receivers, plans for future radio astronomy instruments include arrays of many SIS receivers operating either all in the focal plane of a single radio telescope, or individually on the many antennas of a radio interferometer. For such applications, it is highly desirable to replace mechanically tuned mixers with broadband integrated mixers without mechanical tuners. To produce such mixers will require refinement of present mixer design techniques, most of which use Tucker's threefrequency approximation.

This paper examines the adequacy of three approximations to Tucker's theory: (i) the usual three-frequency approximation, (ii) a fivefrequency approximation, and (iii) a quasi five-frequency approximation. These are compared with a full harmonic-Newton solution [4,5] of Tucker's equations up to the eighth $L O$ harmonic for realistic sis mixer circuits. Use of the approximations greatly reduces the computational effort needed to optimize a mixer design. 


\section{THE APPROXIMATIONS}

In the so-called three-frequency approximation to Tucker's theory, it is assumed, as indicated in Fig. 1(a), that the embedding impedance seen by the junction is finite at the LO frequency $\omega_{p}$, and at $\omega_{u}, \omega_{l}$ and $\omega_{0}$, the upper and lower sideband and intermediate frequencies. At all higher frequencies the junction is short-circuited. This is likely to be a good approximation for junctions with large capacitance. The threefrequency approximation implies a sinusoidal Lo voltage at the junction, for which case Tucker gives closed-form expressions for the coefficients $\mathrm{Y}_{i, j}$ of the mixer's $3 \times 3$ 'small-signal conversion matrix as functions of the pumping parameter $\alpha=\mathrm{eV}_{\mathrm{p}} / \hbar \omega_{\mathrm{p}}$ and DC bias voltage $\mathrm{V}_{0}$.

The quasi five-frequency approximation assumes the same sinusoidal LO voltage waveform as above. However, while the second Lo harmonic $2 \omega_{p}$ is short-circuited at the junction, the second harmonic sidebands $2 \omega_{p} \pm \omega_{0}$ are not. This is depicted in Fig. 1(b). Closed-form expressions for the elements of the $5 \times 5$ small-signal conversion matrix are given by Tucker. While this situation is not easy to implement practically, it is not physically unrealizable, and is expected to be a better approximation to a real mixer than the simple three-frequency approximation.

The full five-frequency approximation allows finite embedding impedances at all frequencies up to $2 \omega_{\mathrm{p}}+\omega_{0}$, but requires the junction to be short-circuited at all higher frequencies. This is depicted in Fig. 1(c). The Lo voltage at the junction contains a second harmonic component, and an iterative algorithm must be used to determine the LO voltage waveform and thence the elements of the $5 \times 5$ small-signal conversion matrix. 


\section{SIMULATIONS}

The three approximations are investigated using hypothetical double sideband SIS mixers at 115 and $345 \mathrm{GHz}$, with the desired embedding impedances at the Lo harmonics and small-signal sideband frequencies. In all the examples, the junction capacitance is tuned out at frequencies $\omega_{2}$ and $\omega_{\mathrm{u}}$ by the source susceptance. The embedding impedance at frequencies $\omega>\omega_{\mathrm{p}}+\omega_{0}$ is either zero or that of the junction capacitance alone, depending on the particular example. The RF source and IF load conductances are assumed equal. These assumptions are consistent with realistic low noise mixer designs [1] with low IF $\left(\omega_{0} \ll \omega_{p}\right)$.

The I-V curve used in the examples is that of the 4-junction array of $\mathrm{Nb} / \mathrm{Al}-\mathrm{Al}_{2} \mathrm{O}_{3} / \mathrm{Nb}$ junctions used in [1], and is shown in Fig. 2. The theoretical equivalence between a series array of junctions and a single junction is shown in [6].

Five values of $\omega_{\mathrm{p}} \mathrm{R}_{\mathrm{N}} \mathrm{C}$ are used: $0.5,1,2,4$, and 50 . In all cases, the pumping parameter $\alpha=\mathrm{eV}_{1} / \hbar \omega_{\mathrm{p}}=1.2$, where $\mathrm{V}_{1}$ is the amplitude of the fundamental component of the Lo voltage at the junction. The junction is. voltage biased at the center of the first photon step below the gap voltage; i.e., $\mathrm{V}_{0}=\mathrm{V}_{\text {gap }}-\hbar \omega / 2 \mathrm{e}$.

The mixer's (equivalent input) noise temperature, (transducer) conversion gain, input return loss, and output impedance are computed for each case as functions of $R_{R F} / R_{N}$, where $R_{R F}$ is the reciprocal of the $R F$ source conductance at the signal frequency and $R_{N}$ is the normal resistance of the junction (or array of junctions). For comparison, the results of the full eight-harmonic analysis are shown on each graph. 


\section{RESULTS}

Ful1 five-frequency approximation

The results of the full five-frequency approximation are shown in Figs. 3(a)-(d) for $115 \mathrm{GHz}$ and $345 \mathrm{GHz}$ mixers. At $115 \mathrm{GHz}$ it is clear that the only significant deviation from the eight-harmonic results is in the gain for the lowest value of $\omega R_{N} C(0.5)$, and that is only a small fraction of a decibel. At $345 \mathrm{GHz}$ there is almost no difference between the full five-frequency approximation and the eight-harmonic results.

Quasi five-frequency approximation

Figures $4(a)-(d)$ show the results of the quasi five-frequency analysis of the same mixers as above. At both 115 and $345 \mathrm{GHz}$, the gain is within a decibel of that computed using eight harmonics. The mixer noise temperatures agree closely for $\omega R_{N} C=50$ and are within about $10 \%$ for $\omega R_{N} C=4$, but for $\omega R_{N} C=0.5$ they differ by as much as $40 \%$. The input return loss and output impedance show minor deviations from the eightharmonic solution.

Three-frequency approximation

In the three-frequency case, the value of $\omega R_{\mathbb{N}} C$ has no effect. This is because all relevant frequencies above the upper sideband are shortcircuited, and we are assuming the junction capacitance is tuned out at the signal and image frequencies. The three-frequency case is thus equivalent to the high- $\omega \mathrm{R}_{\mathrm{N}} \mathrm{C}$ limit of any of the other cases we have considered. The results of Figs. 3 or 4 with $\omega R_{N} C=50$ are, in fact, indistinguishable from those of the three-frequency approximation. 


\section{DISCUSSION}

In an earlier paper [1] we put forward design criteria for SIS mixers for millimeter-wave radio astronomy applications. They should have low noise, conversion gain near unity (to avoid saturation), and a reasonable RF input match. For wide frequency coverage, only doublesideband operation is considered. It was found that these conditions could be met if the mixer operated with equal RF source and IF load conductances, and that the output impedance of the mixer was then large (i.e., the mixer operates as a current source).

The examples here likewise assume equal $R F$ source and IF load conductances, and examine the mixer performance as a function of $R_{R F} / R_{N}\left(R_{R F}\right.$ is the reciprocal of the $R F$ source conductance, and $R_{N}$ is the normal resistance of the junction or array of junctions). As expected, the mixer noise temperature exhibits a broad minimum as $R_{R F} / R_{N}$ is varied (Fig. 3). The (transducer) gain exhibits no minimum, but increases with $R_{R F} / R_{N}$.

At higher frequencies, the minimum noise temperature occurs at larger values of $R_{R F} / R_{N}$. This has an important implication for SIS mixer design: If the same mixer circuit is scaled for use at two different frequencies (i.e., the embedding impedances are the same for the two designs), then the normal resistance of the junction (or array) should be smaller for the higher-frequency mixer. This is discussed in more detail in [1].

In designing a broadband tunerless SIS mixer, the choice of the value of $\omega R_{N} C$ is primarily a tradeoff between noise temperature and useable frequency range; large values of $C$ will obviously limit frequency coverage, while too small an $\omega R_{N} C$ degrades the noise temperature as well as being more difficult to achieve without sacrificing junction quality. It is important, therefore, for the designer to have available a method of mixer analysis which reflects the effect of $\omega R_{N} C$ with sufficient accuracy. From the results in Figs. 3 and 4 , it is clear that the quasi five- 
frequency approximation describes the mixer performance quite well for $\omega R_{\mathrm{N}} \mathrm{C} \geq 4$. For $0.5 \leq \omega \mathrm{R}_{\mathrm{N}} \mathrm{C}<4$, the gain, input return loss, and output impedance are well enough predicted by the quasi five-frequency approximation for most mixer design work, but the mixer noise temperature can be too high by as much as $40 \%$. Figure 5 shows the dependence of the minimum value of $T_{M}$ (with respect to $R_{R F} / R_{H}$ ) on $\omega R_{Y} C$ for each of the methods described here.

It may seem odd that the quasi five-frequency approximation should give quite accurate results for all the mixer parameters except the noise temperature. This is explained by considering the origin and nature of the noise at the output port of the mixer. This noise originates as shot noise from the current flowing in the SIS junction. The action of the local oscillator is, in effect, to amplitude modulate the shot noise produced by the DC current flowing in the junction, thereby generating correlated components at all the sideband frequencies $n \omega_{p} \pm \omega_{0}, n=0$, $1, \ldots$, etc. The mixing action of the time-varying junction conductance converts all these sideband components to the IF, preserving their correlation. The relative phase of each correlated IF component depends on the embedding impedance (including junction capacitance) at its sideband of origin, and on the phases of the harmonics in the LO waveform at the junction. The minimum noise occurs when these correlated IF noise components are phased so as to cancel to the greatest degree. (It is in this way that a classical diode mixer can, in principle, have zero noise temperature while operating with $3 \mathrm{~dB}$ conversion loss and many milliamps of rectified current.) The fact that, in low-noise operation, the SIS mixer has several quite large but strongly correlated output noise components which largely cancel one another, explains the sensitivity of the noise temperature results to the small changes in Lo waveform between the quasi five-frequency and full. five-frequency approximations. (A more detailed discussion of noise conversion in mixers, and additional references are given in [7].) 


\section{REFERENCES}

[1] A. R. Kerr and S.-K. Pan, "Some recent developments in the design of SIS mixers," Int. J. Infrared Millimeter Waves, vol. 11, no. 10, Oct. 1990.

[2] J. R. Tucker, "Quantum limited detection in tunnel junction mixers," IEEE J. of Quantum Electron., vol. QE-15, no. 11, pp. 1234-1258, Nov. 1979.

[3] J. R. Tucker and M. J. Feldman, "Quantum detection at millimeter wavelengths," Rev. Mod. Phys., vol. 57, no. 4, pp. 1055-1113, Oct. 1985.

[4]. C.-Y. E. Tong and R. Blunde1l, "Simulation of superconducting quasiparticle mixer using a five-port model," IEEE Trans. Microwave Theory Tech., vol. MTT-38, no. 10, pp. 1391-1398, Oct. 1990.

[5] S. Withington and P. Kennedy, "Numerical procedure for simulating the large-signal quantum behavior of superconducting tunnel-junction circuits,": Proc. IEE, part G, vol. 138, no. 1, pp. 70-76, Feb. 1991.

[6] M. J. Feldman and S. Rudner, "Mixing with SIS arrays," Reviews of Infrared \& Millimeter Waves, (Plenum, New York), vol. 1, p. 47-75, 1983.

[7] D. N. Held and A. R. Kerr, "Conversion loss and noise of microwave and millimeter-wave mixers: Part 1 - Theory, " IEEE Trans. Microwave Theory Tech., vol. MTT-26, pp. 49-55, Feb. 1978. 


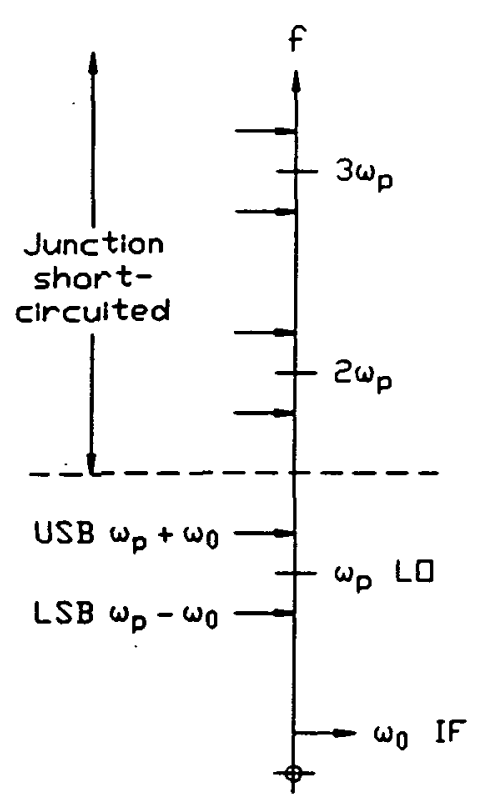

3-FREQ. APPROX.

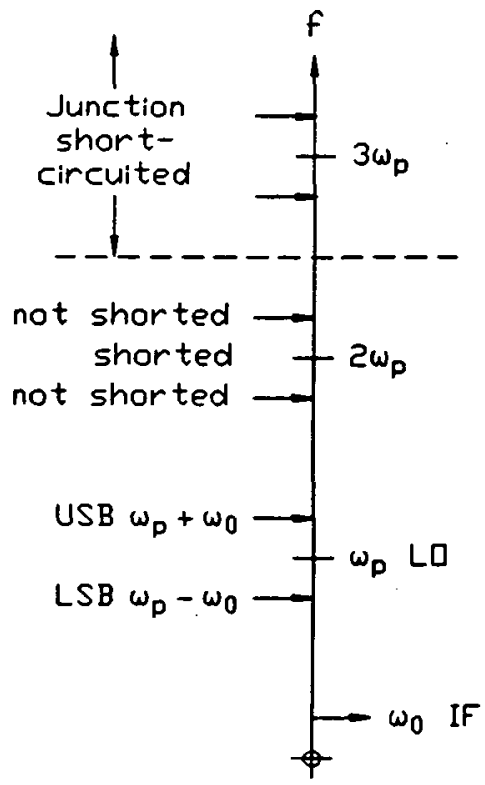

QUASI 5-FREQ. APPRIX.

(b)

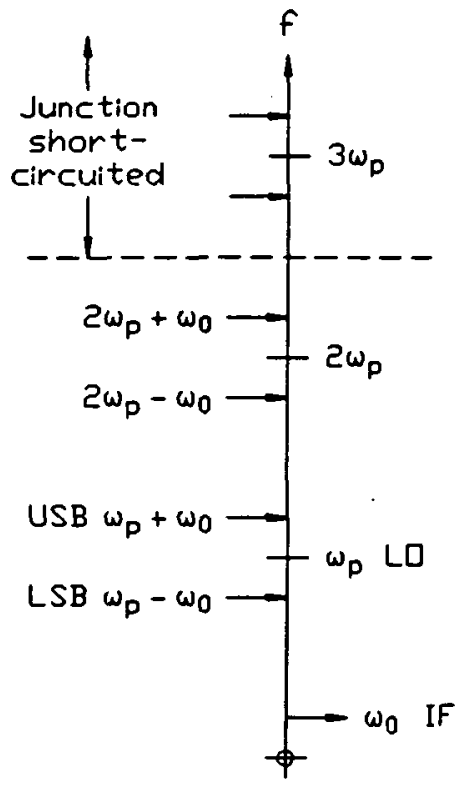

5-FREQ. APPRDX.

(C)

Fig. 1. Embedding impedance diagram indicating which frequencies are short-circuited for the three approximations: (a) three-frequency, (b) quasi five-frequency, and (c) full five-frequency.

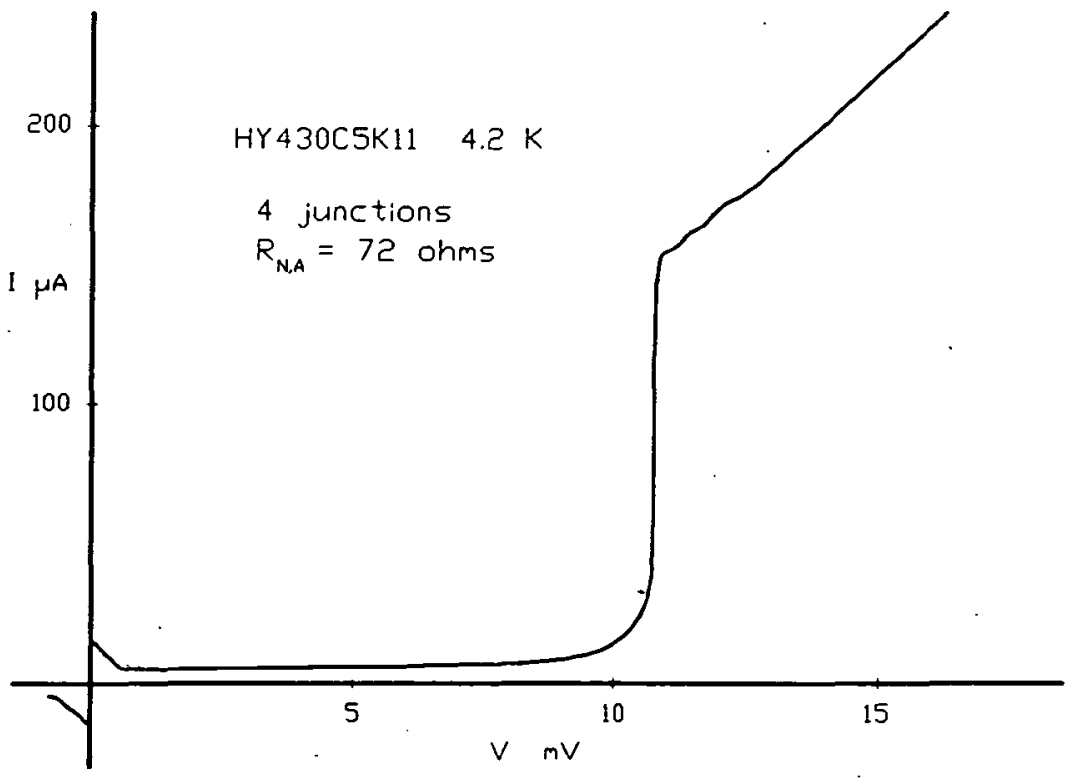

Fig. 2. I-V curve used in the simulations. This curve is for a four-junction array of Hypres $\mathrm{Nb} / \mathrm{Al}-\mathrm{Al}_{2} \mathrm{O}_{3} / \mathrm{Nb}$ junctions at $4.2 \mathrm{~K}$, as used in [1]. 


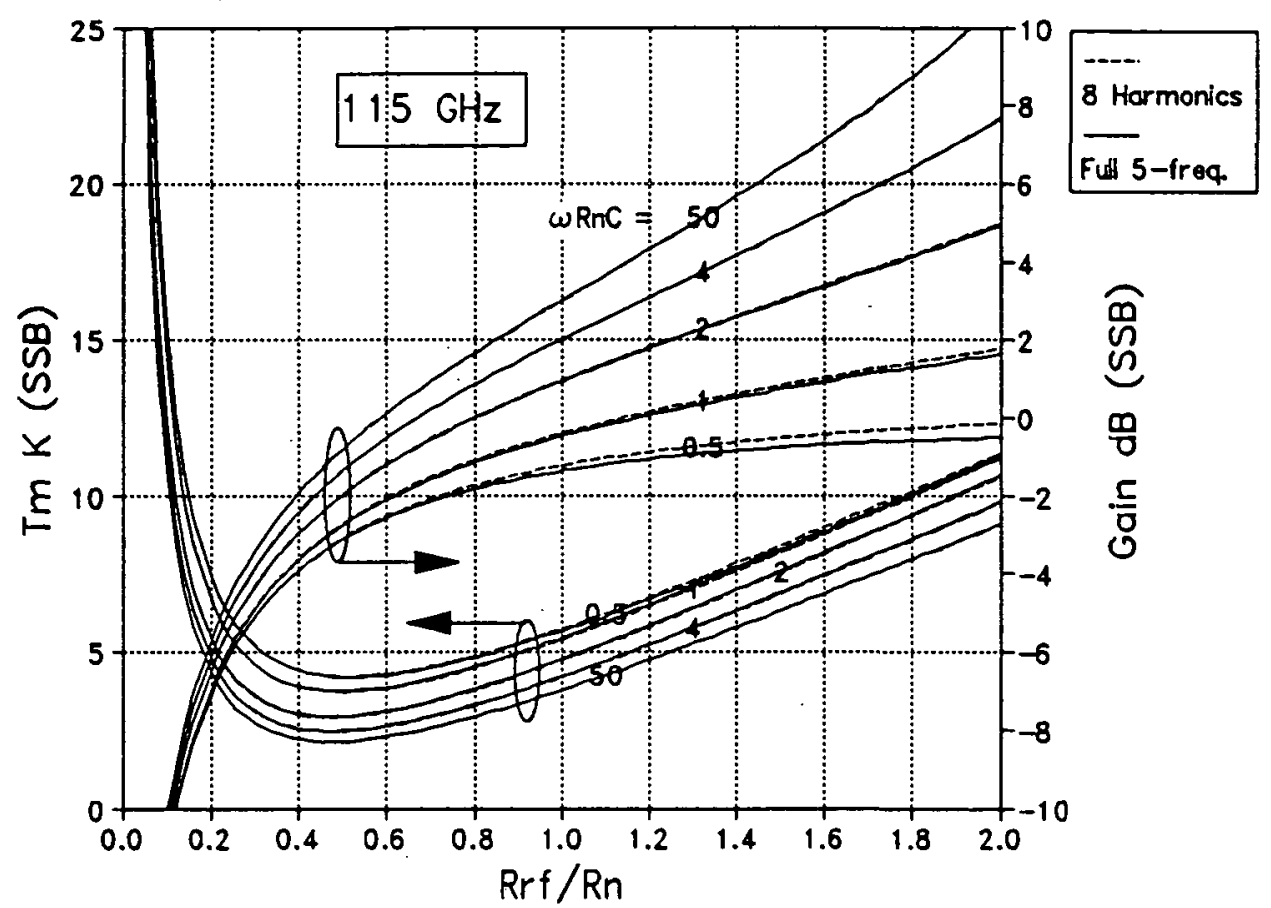

Fig. 3(a). Noise temperature and conversion gain as functions of $R_{R F} / R_{N}$ for a $115 \mathrm{GHz}$ SIS mixer, computed using the full five-frequency approximation (solid line) and with eight LO harmonics (broken line). Results are shown for $\omega R_{N} C=0.5,1,2,4$, and 50 .

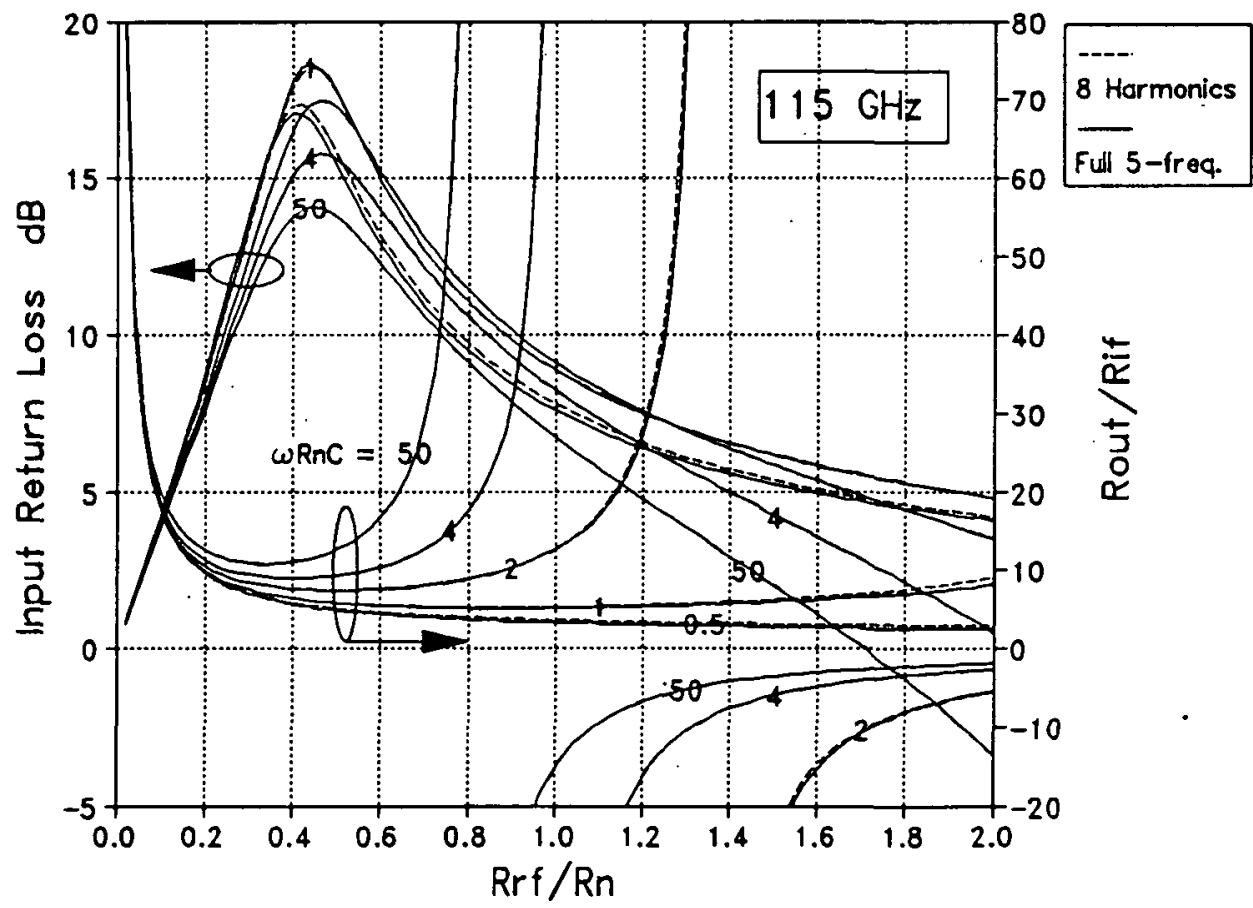

Fig. 3(b). Input return loss and output impedance as functions of $R_{R F} / R_{N}$ for a $115 \mathrm{GHz}$ SIS mixer, computed using the full five-frequency approximation (solid line) and with eight LO harmonics (broken line). The output resistance is normalized to the IF load resistance $R_{\mathrm{IF}}$. Results are shown for $\omega R_{N} C=0.5,1,2,4$, and 50 . 


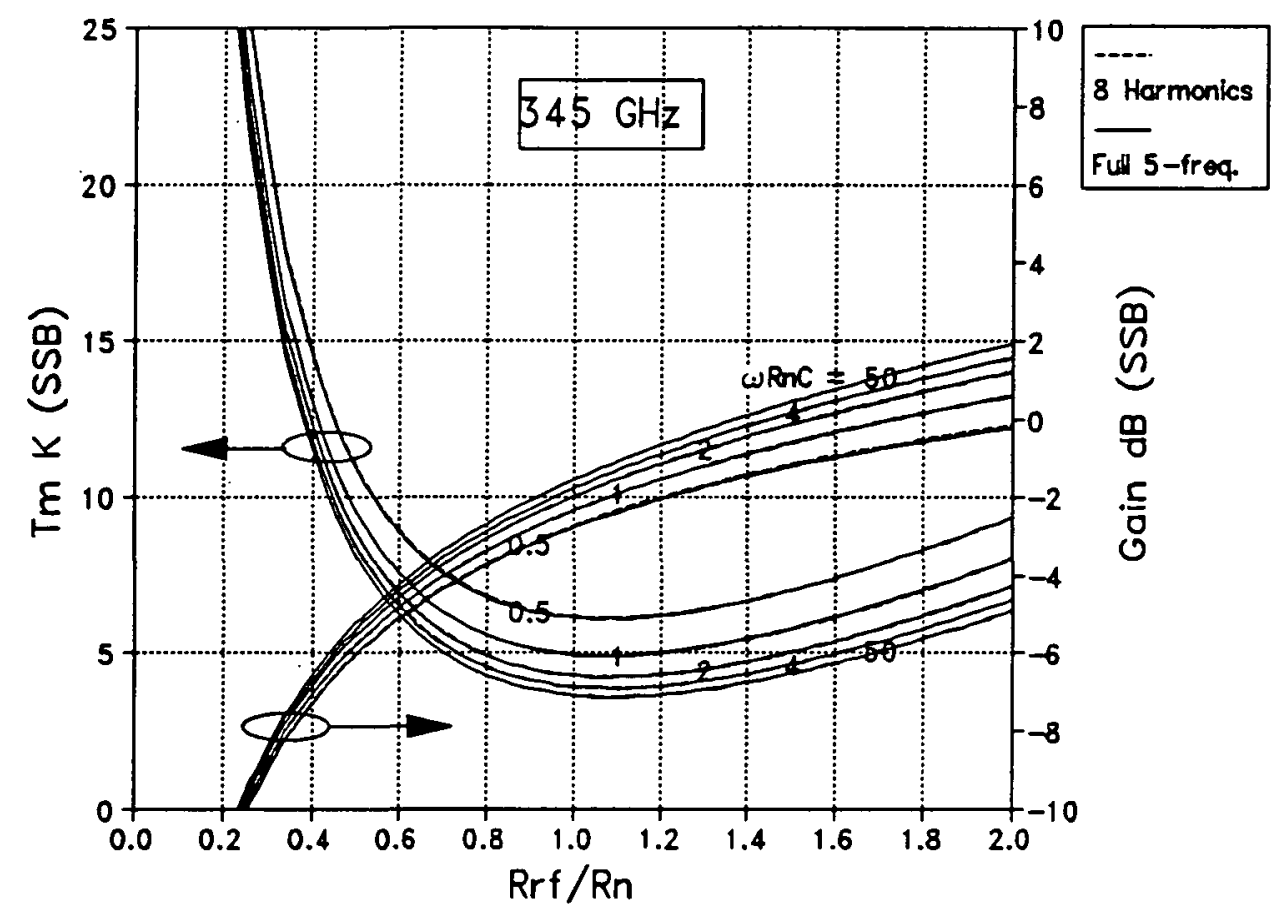

Fig. 3(c). Noise temperature and conversion gain as functions of $R_{R F} / R_{N}$ for a $345 \mathrm{GHz}$ SIS mixer, computed using the full five-frequency approximation (solid line) and with eight LO harmonics (broken lines - almost obscured by the solid lines). Results are shown for $\omega R_{N} C=0.5,1,2,4$, and 50.

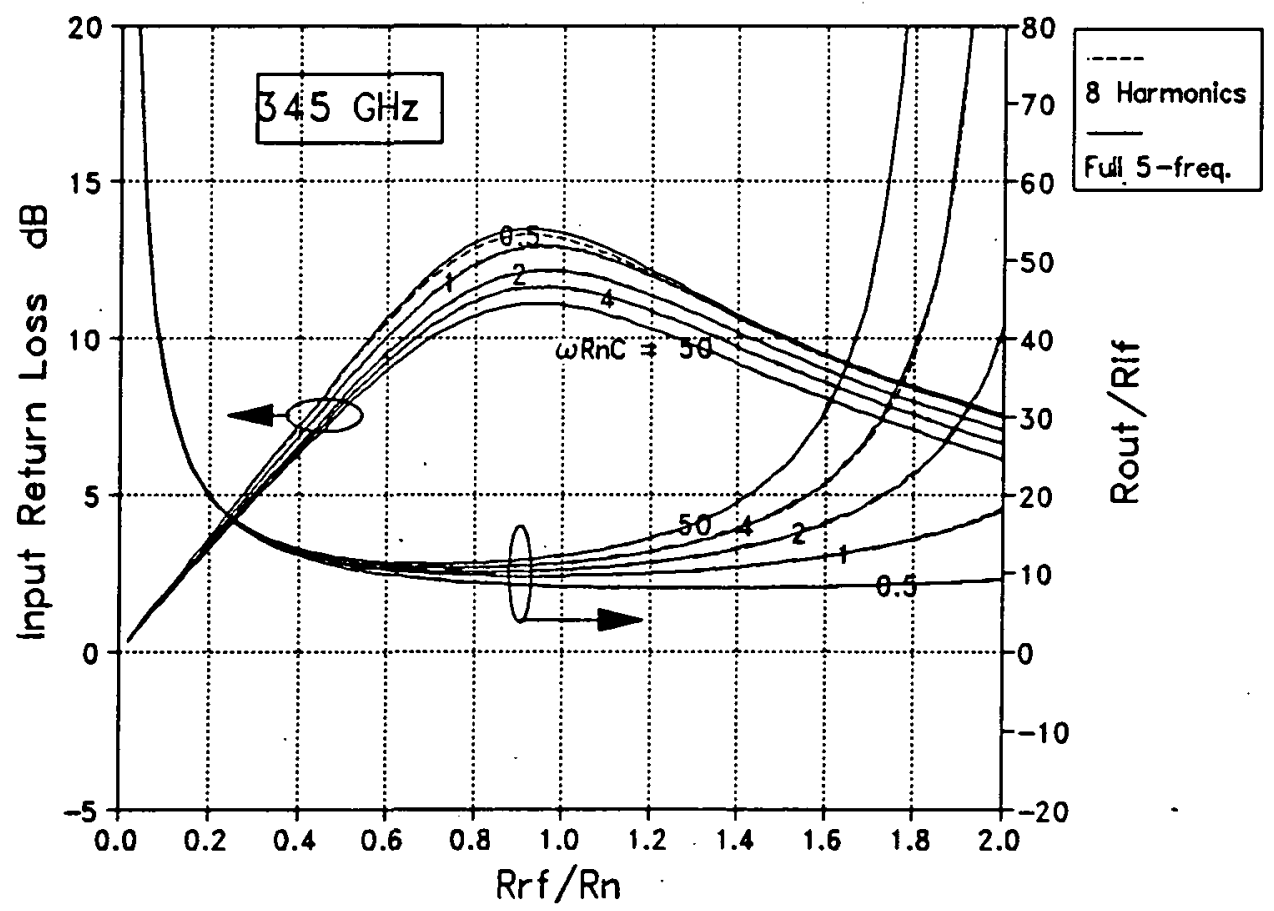

Fig. 3(d). Input return loss and output impedance as functions of $R_{\mathrm{RF}} / R_{\mathrm{N}}$ for a $345 \mathrm{GHz}$ SIS mixer, computed using the full five-frequency approximation (solid line) and with eight LO harmonics (broken lines - almost obscured by the solid lines). The output resistance is normalized to the IF load resistance $R_{\mathrm{IF}}$. Results are shown for $\omega R_{N} C=0.5,1,2,4$, and 50 . 


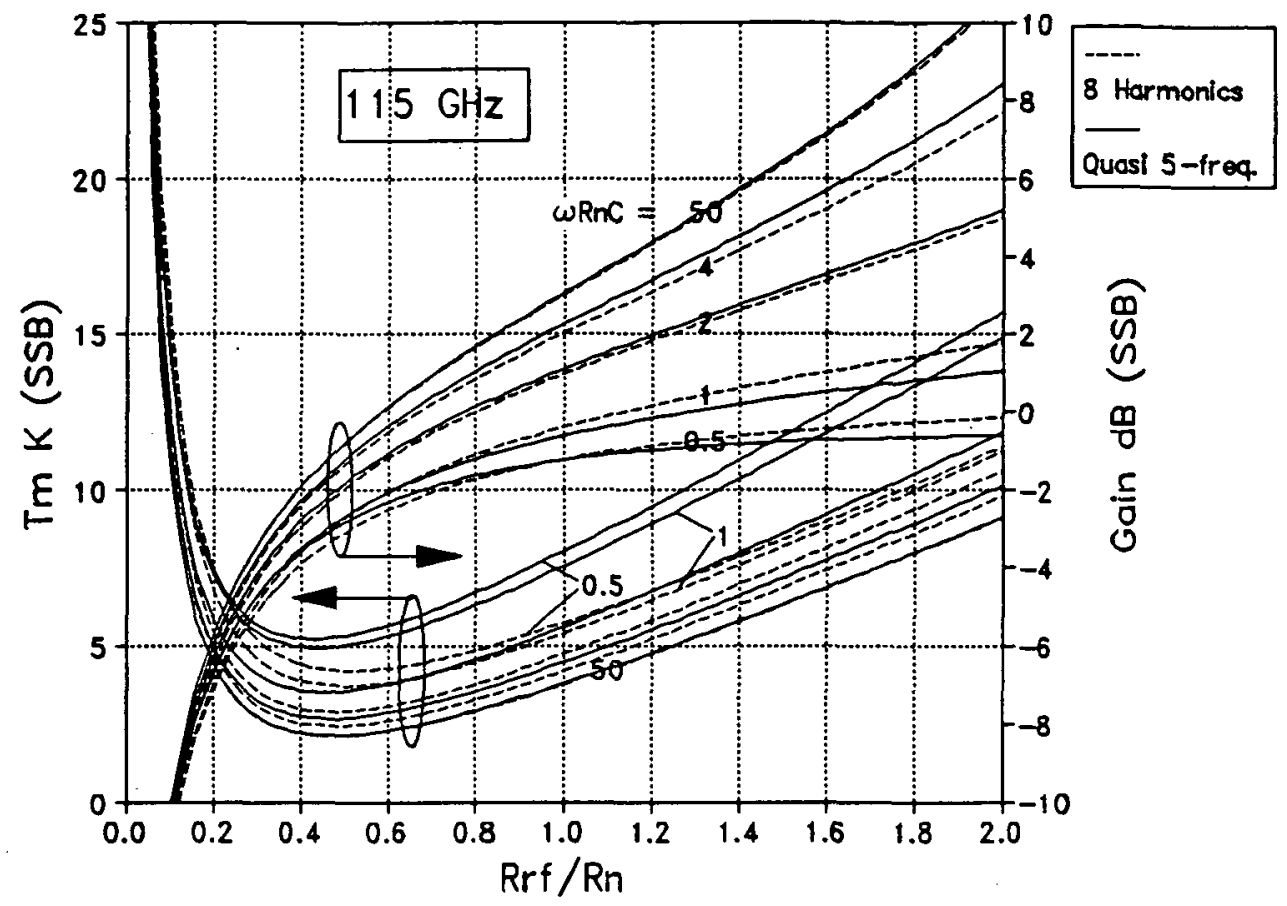

Fig. 4(a). Noise temperature and conversion gain as functions of $R_{R F} / R_{N}$ for a $115 \mathrm{GHz}$ SIS mixer, computed using the quasi five-frequency approximation (solid line) and with eight LO harmonics (broken line). Results are shown for $\omega R_{N} C=0.5,1,2,4$, and 50 .

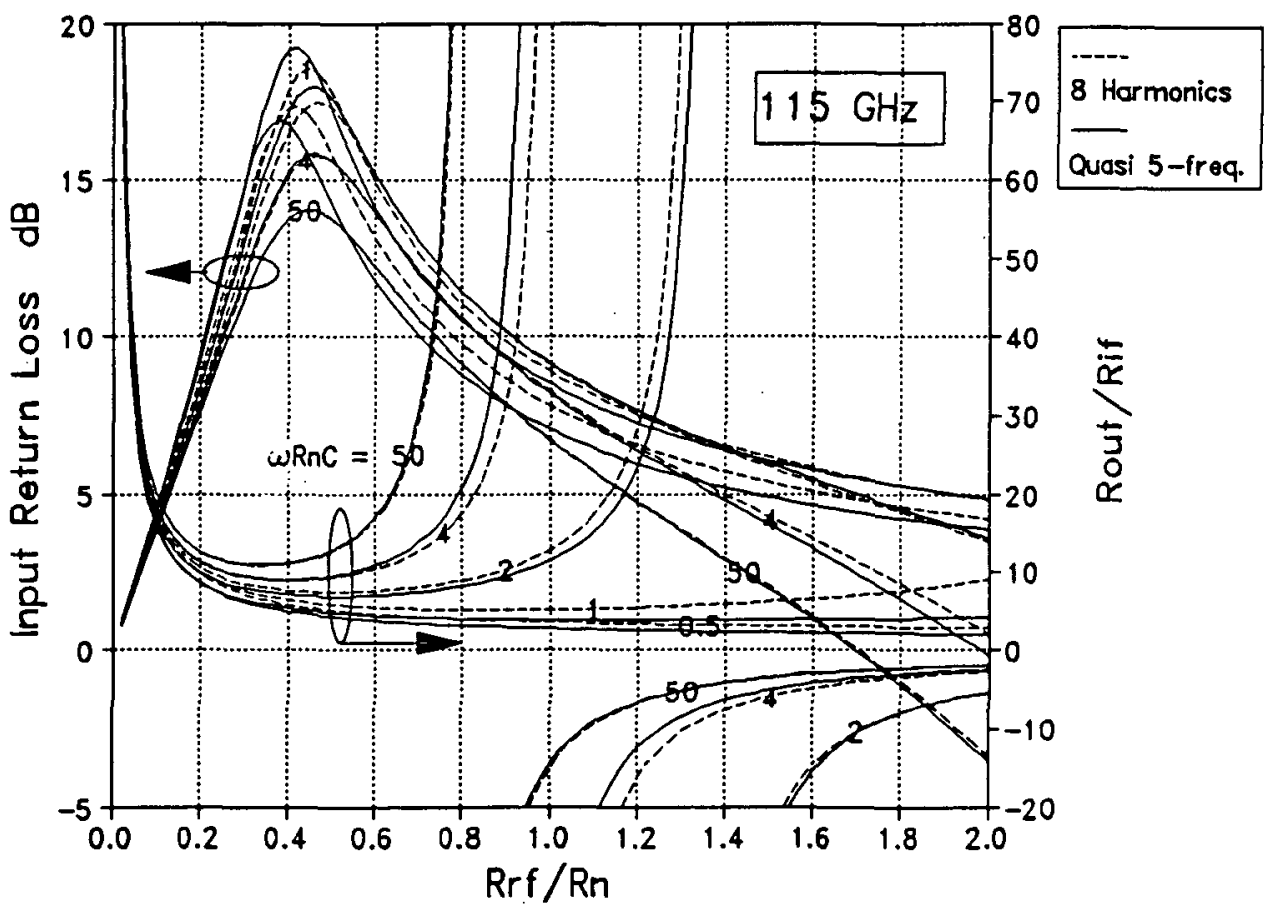

Fig. 4(b). Input return loss and output impedance as functions of $R_{\mathrm{RF}} / R_{\mathrm{N}}$ for a $115 \mathrm{GHz}$ SIS mixer, computed using the quasi five-frequency approximation. (solid line) and with eight LO harmonics (broken line). The output resistance is normalized to the IF load resistance $R_{\mathbb{I F}}$. Results are shown for $\omega R_{N} C=0.5,1,2,4$, and 50 . 


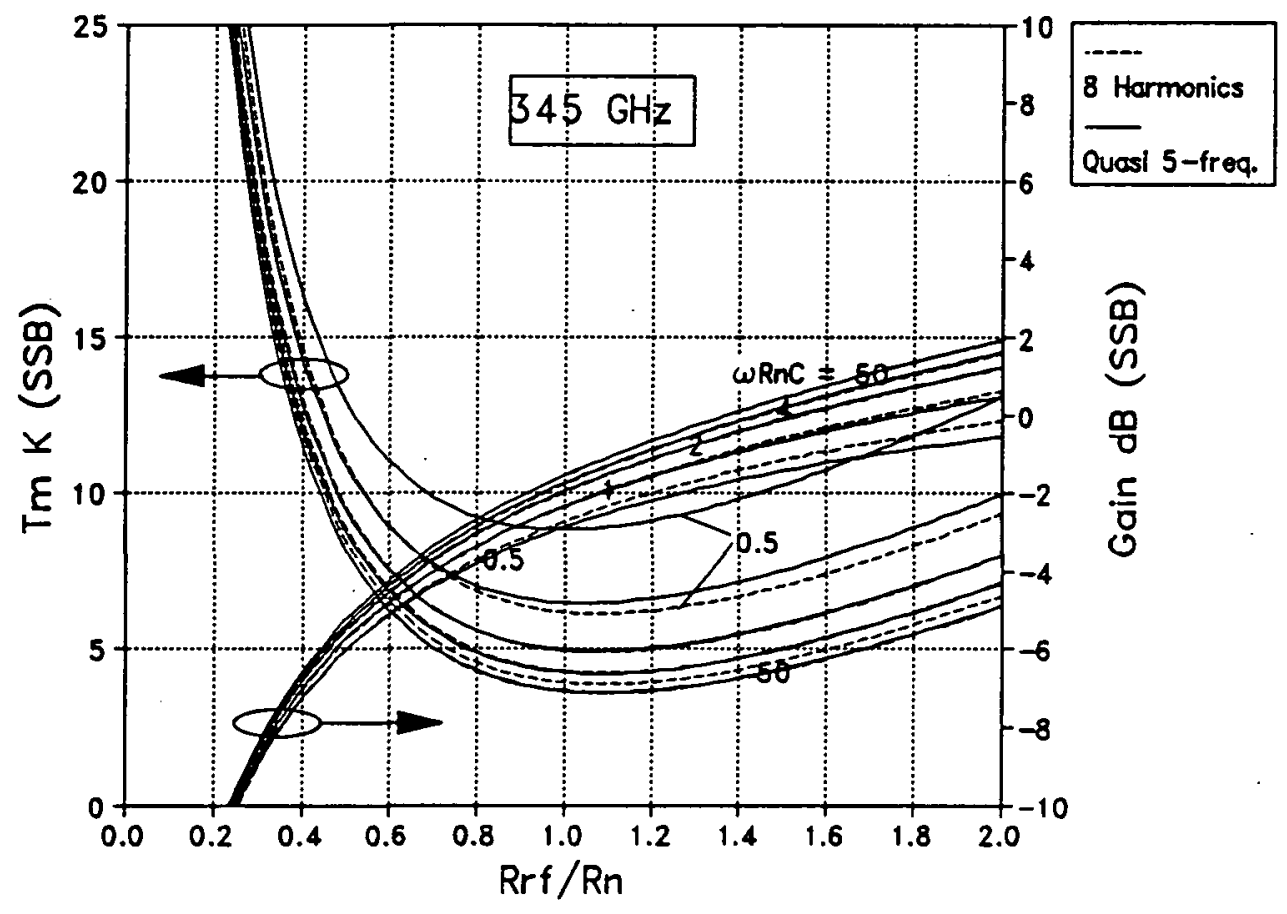

Fig. $4(c)$. Noise temperature and conversion gain as functions of $R_{R F} / R_{N}$ for a $345 \mathrm{GHz}$ SIS mixer, computed using the quasi five-frequency approximation (solid line) and with eight LO harmonics (broken line). Results are shown for $\omega R_{N} C=0.5,1,2,4$, and 50 .

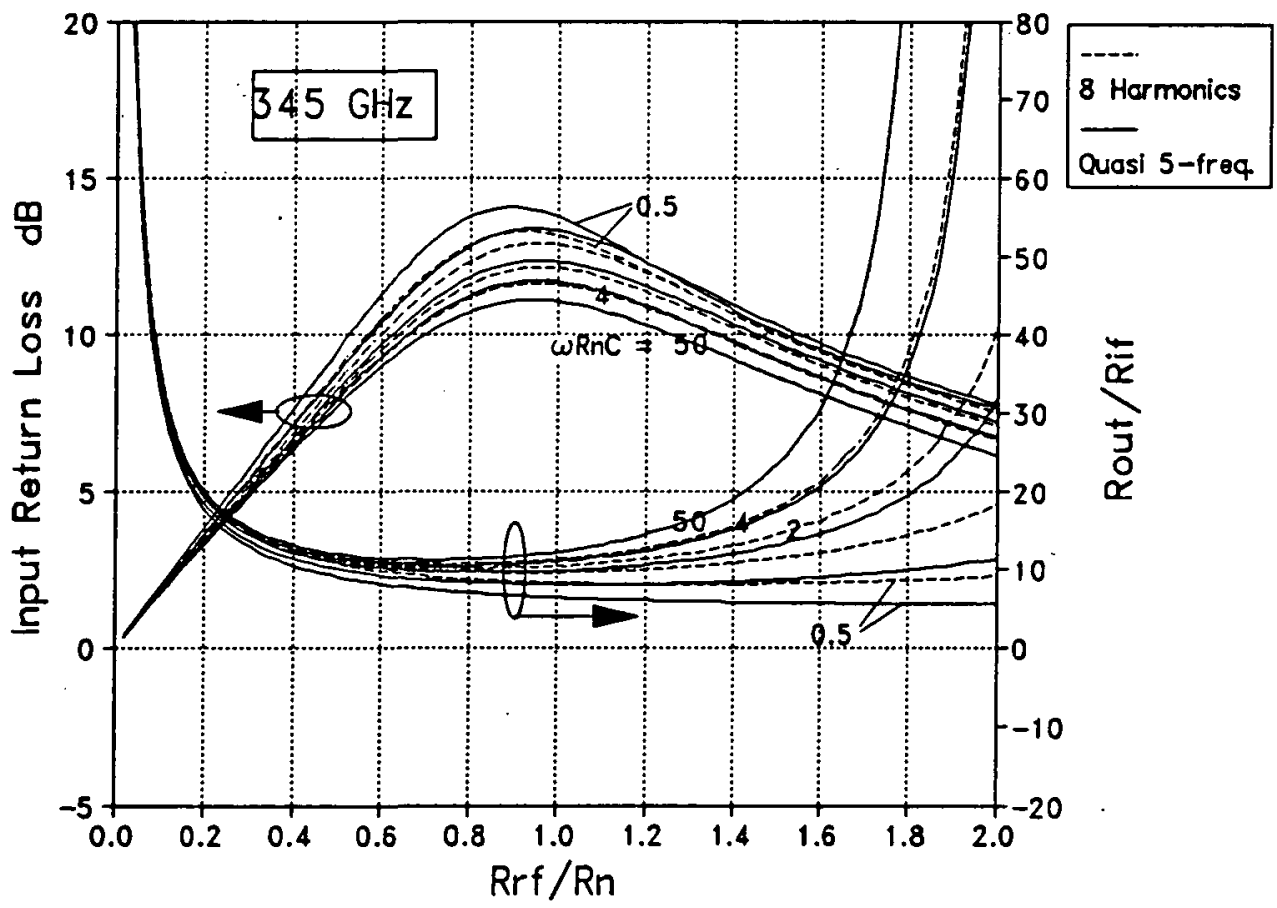

Fig. 4(d). Input return loss and output impedance as functions of $R_{\mathrm{RF}} / \mathrm{R}_{\mathrm{N}}$ for a $345 \mathrm{GHz}$ SIS mixer, computed using the quasi five-frequency approximation (solid line) and with eight LO harmonics (broken line). The output resistance is normalized to the IF load resistance $R_{I F}$. Results are shown for $\omega R_{N} C=0.5,1,2,4$, and 50 . 


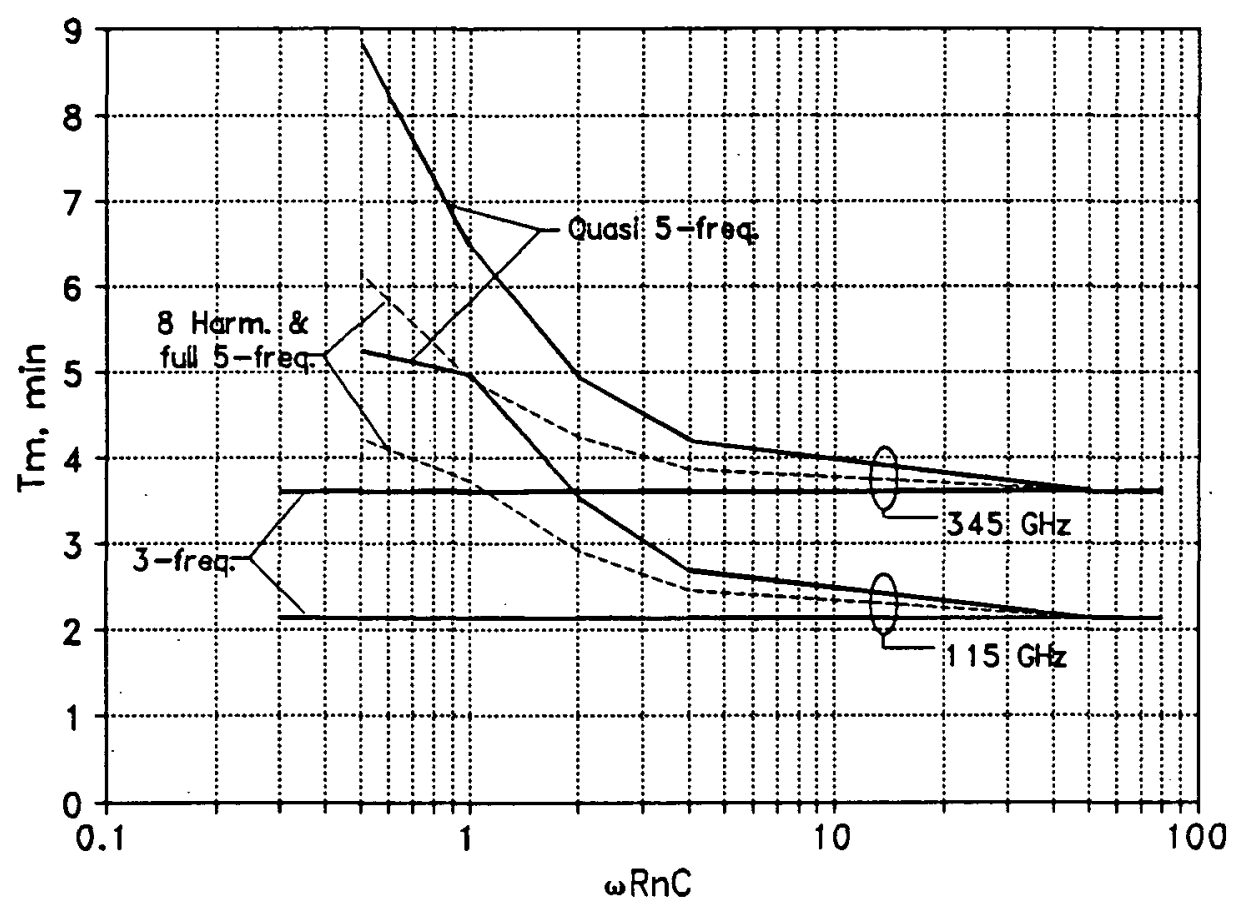

Fig. 5. Minimum mixer noise temperature as a function of $\omega R_{N} C$ for: (i) the full eight-harmonic and full 5-frequency solutions (broken line), (ii) the quasi 5-frequency approximation (solid line), and (iii) the 3-frequency approximation (horizontal lines). 